\title{
Zinc-binding metallothioneins are key modulators of IL-4 production by basophils
}

Tsukasa Ugajin, ${ }^{\mathrm{a}, \mathrm{b}}$ Keigo Nishida, ${ }^{\mathrm{b}, \mathrm{c}}$ Satoru Yamasaki, ${ }^{\mathrm{b}}$ Junko Suzuki, ${ }^{\mathrm{d}}$ Masaharu Mita, ${ }^{\mathrm{e}}$ Masato Kubo, ${ }^{\dagger}$ Hiroo Yokozeki, ${ }^{a}$ and Toshio Hirano ${ }^{g}$

${ }^{a}$ Department of Dermatology, Graduate School, Tokyo Medical and Dental University, 1-5-45 Yushima, Bunkyo-ku, Tokyo 113-8519, Japan; 'baboratory for Homeostatic Network, RIKEN Research Center for Integrative Medical Sciences (IMS), 1-7-22 Suehiro-cho,

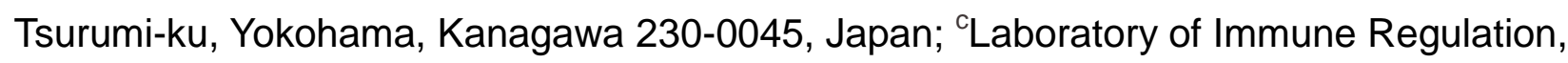
Faculty of Pharmaceutical Sciences, Suzuka University of Medical Science, 3500-3 Minamitamagaki-cho, Suzuka, Mie 513-8670, Japan; ${ }^{d}$ Center for Environmental Risk Research, National Institute for Environmental Studies, 16-2 Onogawa, Tsukuba, Ibaraki 305-8506, Japan; 'Laboratory Animal Research Center, School of Pharmacy, Kitasato University, 5-9-1 Shirokane, Minato-ku, Tokyo 108-8641, Japan; ${ }^{\dagger}$ Division of Molecular Pathology, Research Institute for Biomedical Science, Tokyo University of Science, 2641 Yamazaki, Noda, Chiba 278-8510, Japan; and 'OOsaka University, 1-1 Yamada-oka, Suita, Osaka 565-0871, Japan 
Running head: Role of zinc-metallothioneins in basophil function

Address correspondence and reprint requests:

Toshio Hirano, MD, PhD, Osaka University, 1-1, Yamada-oka, Suita, Osaka 565-0871, Japan

Tel: +81-6-6879-7000 Fax: +81-6-6879-7006 E-mail: hirano@hq.osaka-u.ac.jp

Keigo Nishida, PhD, Laboratory for Homeostatic Network, RIKEN Research Center for Integrative Medical Sciences (IMS), 1-7-22, Suehiro, Tsurumi, Yokohama, Kanagawa 230-0045, Japan

Tel: +81-45-503-7075 Fax: +81-45-503-9688 E-mail: nishida@rcai.riken.jp

Number of pages: 32 ; Number of figures: $5+3$ supplemental; Number of references: 35 
Abbreviations used in this paper: BMBA, bone marrow-derived basophil; $\mathrm{Ca}$, calcium;

CaN, calcineurin; CsA, cyclosporin A; FceRI, Fc epsilon receptor 1; MT, metallothionein;

NFAT, nuclear factor of activated T-cells; PI3K, phosphoinositide 3-kinase; PKC, protein

kinase C; siRNA, short interfering RNA; Th2, T helper 2; TNP, trinitrophenyl; Zip,

ZRT/IRT-related protein; Zn, Zinc; ZnT, Zn transporter. 


\section{Introduction}

Zinc (Zn), an essential trace element, forms a structural component of many proteins, including enzymes in cellular signaling pathways and transcription factors, and it is essential for their biological activities (Fukada et al., 2011; Hirano et al., 2008). Defects in Zn homeostasis cause growth retardation, immunodeficiency, and other diseases (Prasad, 1995). Zn homeostasis is tightly controlled by Zip/SIc39 and ZnT/SIc30 family members, Zn transporters that increase or decrease the intracellular $\mathrm{Zn}$, and by $\mathrm{Zn}$-binding proteins such as metallothioneins (MTs) (Andrews, 2001; Palmiter, 2004).

MTs are low molecular weight $(\sim 7 \mathrm{kDa})$ proteins that exhibit a high affinity for divalent heavy metal ions. MTs are grouped into four classes (I-IV) (Thirumoorthy et al., 2011). MT-I and MT-II, which are nearly ubiquitous, are induced by metals and inflammatory stress signals. MT-III is predominantly expressed in neurons and glia. MT-IV is expressed in differentiating stratified squamous epithelial cells (Palmiter, 1998). MTs are reported to assist in the detoxification of heavy metals such as mercury and cadmium, in the homeostasis of essential metals such as copper and zinc, and in protecting cells from oxidative stress caused by hydroxyl free radicals and nitric oxide (Thirumoorthy et al., 2011). MTs also function to buffer $\mathrm{Zn}$, thereby regulating the cytosolic $\mathrm{Zn}$ ion concentration. However, it is not known whether MTs regulate intracellular signal transduction. 
In mast cells, aggregation of the high-affinity IgE receptor Fc\&RI initiates the $\mathrm{Zn}$ wave, a phenomenon in which free $\mathrm{Zn}$ is released from the endoplasmic reticulum (ER) (Yamasaki et al., 2007). The Zn wave positively modulates NF-kB's DNA-binding activity, thereby regulating the mast cells' cytokine production downstream of Fc\&RI (Yamasaki et al., 2012). In addition, the ZnT-family member ZnT5 is required for the Fc\&RI-induced translocation of PKC- $\beta$ to the plasma membrane, and therefore, for the NF-kB-dependent cytokine production by mast cells (Nishida et al., 2009). These results indicate that $\mathrm{Zn}$ acts as an intracellular signaling molecule in mast cells, and that $\mathrm{Zn}$ is essential for the FceRI-induced cytokine production by mast cells.

Basophils represent less than $1 \%$ of the peripheral blood leukocytes. Like mast cells, they express cell-surface Fc\&RI and release cytokines and other chemical mediators after Fc\&RI cross-linking (Galli, 2000; Karasuyama and Yamanishi, 2014). Under normal physiological conditions, basophils circulate in the blood while mast cells reside in peripheral tissues. Basophils infiltrate peripheral tissue in response to allergic inflammation, such as in bronchial asthma and atopic dermatitis (Macfarlane et al., 2000; Plager et al., 2006). While basophils have long been neglected in immunological studies because of their small numbers and their morphological similarity to mast cells, they are now recognized as a major source of Th2-promoting cytokines, such as IL-4 and TSLP (Schroeder et al., 2001; Seder et al., 1991; Sokol et al., 2008; Voehringer et al., 2004). However, the molecular mechanisms of basophil IL-4 production have not been fully elucidated. 
In the present study, we hypothesized that Zn plays a role in the basophils' IL-4 production. We first examined the expression profiles of $\mathrm{Zn}$-controlling molecules in mouse basophils, and found that FceRI stimulation significantly induced the Mt-1 and Mt-2 genes. We then demonstrated that MTs were required for the Fc\&RI-induced basophil production of IL-4. We also found that MTs were required to maintain the proper intracellular Zn concentration and to regulate calcineurin $(\mathrm{CaN})$ phosphatase activity, thus impacting the FceRI-induced nuclear factor of activated T-cell (NFAT)-dependent IL-4 production. Our findings indicate that MT-dependent Zn regulation is essential for basophil IL-4 production. 


\section{Material and methods}

\subsection{Mice}

C57BL/6 mice (8-10 weeks old) were purchased from CREA (Tokyo, Japan). MT-I/II(-/-) C57BL/6 mice were kindly provided by Dr. Chiharu Tohyama (The University of Tokyo, Japan). All experiments were conducted according to RIKEN's Guidelines for Animal Use and Experimentation.

\subsection{Antibodies and reagents}

Purified rat anti-mouse CD16/CD32 (2.4G2), biotinylated monoclonal antibodies (mAbs) specific for CD49b (Dx5), purified rat anti-mouse IgE, and purified rat IgG1 kappa (the isotype-matched control) were from BD Pharmingen (San Diego, CA, USA).

FITC-conjugated anti-CD49b (Dx5), PE-anti-mouse CD123, and PE-conjugated anti-FcERI $\alpha$ (MAR1) were purchased from eBioscience (San Diego, CA, USA); anti-PKC- $\beta$ and anti-actin antibodies (Abs) from Santa Cruz Biotechnology (Santa Cruz, CA, USA); anti-AKT and anti-phospho AKT Abs from Cell Signaling Technology (Danvers, MA, USA); and anti-NFATc1 mAb (7A6) from Thermo Scientific (Rockford, IL, USA). Anti-mouse mast cell protease (mMCP)-8 mAb (TUG8) was established by the first author (Ugajin et al., 2009). Cyclosporin A, Go6976, and wortmannin were from Calbiochem (San Diego, CA, USA). 


\subsection{Cell preparation and culture}

Bone marrow-derived basophils (BMBAs) were prepared by culturing bone marrow cells in the presence of rIL-3 for 10 days, followed by isolating the CD49b+ cells using a magnetically activated cell sorter (MACS) system as described previously (Ugajin et al., 2011). To prepare primary basophils, freshly isolated bone marrow cells were MACS-enriched for $\mathrm{CD} 49 \mathrm{~b}^{+}$cells, and $\mathrm{CD} 49 \mathrm{~b}^{+} \mathrm{CD} 123^{+}$cells were isolated using a FACSVantage (BD Biosciences, Mountain View, CA, USA). The purity of the sorted cells was $>98 \%$.

\subsection{Stimulation of basophils}

BMBAs $\left(1 \times 10^{6}\right.$ cells $/ \mathrm{ml}$ ) were sensitized with $1 \mu \mathrm{g} / \mathrm{ml}$ anti-TNP (trinitrophenyl)-lgE (IGEL-b4) in the absence of IL-3 for $6 \mathrm{~h}$ at $37^{\circ} \mathrm{C}$, washed, resuspended in Tyrode's buffer, and stimulated with the indicated concentrations of TNP-OVA (Bioresearch Technologies, Novato, CA, USA) for the indicated times. Ex vivo lgE-mediated stimulation of basophils was performed as described previously (Charles et al., 2009).

\subsection{Degranulation assay}

The degranulation assay was described previously (Nishida et al., 2009). In brief, BMBAs $\left(1 \times 10^{6} \mathrm{cells} / \mathrm{ml}\right)$ were sensitized with $1 \mu \mathrm{g} / \mathrm{ml}$ anti-TNP $\mathrm{lgE}$ in the absence of IL-3 for $6 \mathrm{~h}$, followed by stimulation with $100 \mathrm{ng} / \mathrm{ml}$ TNP-OVA in Tyrode's buffer for $30 \mathrm{~min}$. The cells 
were centrifuged at $200 \times \mathrm{g}$ for $5 \mathrm{~min}$, and the pellets solubilized with $1 \%$ Triton $\mathrm{X}-100$ in Tyrode's buffer. The enzymatic activity of $\beta$-hexosaminidase in the supernatants and solubilized cell pellets was then measured with $p$-nitrophenyl $N$-acetyl- $\beta$-d-glucosaminide (Sigma-Aldrich) in $0.1 \mathrm{M}$ sodium citrate. The degranulation level was determined by measuring the release of $\beta$-hexosaminidase.

\subsection{Measurement of IL-4}

The IL-4 in cell-culture supernatants was measured with an ELISA kit (R\&D, Minneapolis, MN, USA).

\subsection{Immunoblotting}

Cells were lysed in buffer containing 1\% NP-40 and a protease inhibitor cocktail (Active Motif, Carlsbad, CA, USA). Total cell lysates were separated by SDS-PAGE using a 5-20\% polyacrylamide gel, transferred to a PVDF membrane, and subjected to immunoblotting with the indicated Abs and HRP-conjugated species-specific secondary Abs. The PVDF membranes were exposed to RX film (Fujifilm, Tokyo, Japan). Densitometric analysis was performed by LAS-1000 (Fujifilm).

\subsection{Calcineurin phosphatase assay}

Calcineurin $(\mathrm{CaN})$ phosphatase activity was measured using a Calcineurin Phosphatase 
Assay Kit (Enzo Life Sciences, Plymouth Meeting, PA, USA) according to the manufacturer's instructions. Briefly, BMBAs pretreated with (background) or without $10 \mu \mathrm{M}$ cyclosporin $\mathrm{A}$ (CsA, a $\mathrm{CaN}$ inhibitor) for 20 min were homogenized with a glass homogenizer in $0.25 \mathrm{M} \mathrm{HES}$ buffer and centrifuged at $1000 \times \mathrm{g}$ for $10 \mathrm{~min}$. The supernatants were incubated with $0.75 \mathrm{mM}$ RII phosphopeptide substrate for $10 \mathrm{~min}$. Free phosphate released into the supernatants was determined by a malachite green assay. Phosphate released by $\mathrm{CaN}$ was calculated after background subtraction.

\subsection{Flow cytometric analysis}

Single-cell suspensions were treated with $2.4 \mathrm{G} 2$ and normal rat serum, and were stained with the indicated Abs. Cells were analyzed using a FACSCalibur (BD Biosciences, Mountain View, CA, USA), and data were analyzed with CellQuest software (BD Biosciences).

\subsection{Zn quantification by flow cytometry}

BMBAs were treated with $0.5 \mu \mathrm{M}$ Newport green DCF diacetate (Invitrogen, Carlsbad, CA, USA) for 30 min at $37^{\circ} \mathrm{C}$. The fluorescence intensity of the BMBAs was measured using a FACS Caliber (BD Biosciences, Mountain View, CA, USA). The data were analyzed with CellQuest software (BD Biosciences). 


\subsection{Calcium influx measurement}

IgE-sensitized BMBAs were incubated with $10 \mu \mathrm{M}$ Indo-1-AM, a cell-permeable Ca-sensor dye (Invitrogen), and Pluronic F-127 (Sigma-Aldrich) for 30 min at $37^{\circ} \mathrm{C}$. The cells were washed and stimulated with antigen. Cells were monitored with a BD LSRII flow cytometer (BD Biosciences). Values were plotted as the ratio of $\mathrm{Ca}^{2+}$-bound Indo-1 $(\mathrm{FL} 5) / \mathrm{Ca}^{2+}$ to free Indo-1 (FL4).

\subsection{Confocal microscopy}

For mMCP-8 staining, cytospin preparations were fixed in methanol and treated with blocking solution containing $10 \%$ normal goat serum, $0.01 \%$ Triton $\mathrm{X}-100$, and $2.4 \mathrm{G} 2$. The preparations were then incubated with an Alexa 488-conjugated anti-mMCP-8 mAb. For NFAT2 and PKC- $\beta$ staining, cells were fixed with $4 \%$ paraformaldehyde before spinning. Cytospin preparations were permeabilized with Perm Buffer (BD Biosciences) containing $1 \%$ BSA and 2.4G2. The preparations were incubated with anti-NFAT2 mAb or anti-PKC- $\beta$ $\mathrm{mAb}$, followed by Alexa488-conjugated anti-mouse IgG (Invitrogen) or Alexa488-conjugated anti-rabbit IgG (Invitrogen), respectively. The cells were examined using a TCS SL system (Leica, Bensheim, Germany).

\subsection{Real-time PCR analysis}

After preparing total cell RNA, first-strand cDNA was synthesized using reverse 
transcription with oligo-dT primers. Gene expression was measured using SYBR Green Dye (Applied Biosystems, Foster, CA, USA) with a real-time PCR system, and was normalized to Gapdh. The following primers (forward and reverse, respectively) were used for both PCR reactions: for Gapdh, 5'-TTCACCACCATGGAGAAGGCCG-3' and 5'-GGCATGGACTGTGGTCATGA-3'; for Mt1, 5'-GCCTGCAAGAACTGCAAGTG-3' and 5'-ATAGGAAGACGCTGGGTTGG-3'; for Mt2, 5'-TGGAGAACGAGTCAGGGTTG-3' and 5'-TCCTGCAAGAAAAGCTGCTG-3'; for mll-4, 5'-ACAGGAGAAGGGACGCCAT-3' and 5'-GAAGCCCTACAGACGAGCTCA-3'.

\subsection{Plasmid construction and transfection}

The coding region of the mouse Mt2 gene was isolated from a cDNA library of the C57BL/6 mouse. N-terminally HA-tagged Mt-2 cDNA was inserted into a pCAGI puro expression vector (a gift from Dr. H. Niwa, RIKEN, CDB, Japan). BMBAs were transfected with plasmid vectors or siRNA (Thermo Fisher Scientific, MA, USA) using a two-step electroporator, CUY21ProVitro (Nepa Gene, Chiba, Japan). For electroporation, $1 \times 10^{6}$ BMBAs were re-suspended in $100 \mu \mathrm{l}$ of OPTI-MEM, and $12.5 \mu \mathrm{g}$ of plasmids or $400 \mathrm{pmol}$ of siRNA were added. Electroporation was carried out as described previously (Yamasaki et al., 2012).

\subsection{Luciferase assay}

BMBAs were transfected with the luciferase reporter plasmid (pGL4-IL-4) (Kubo et al., 
1994), the renilla reporter plasmid (phRL-TK), and the CAG-puro-HA-MT2 or empty CAG vector. The cells were collected after stimulation and were analyzed using the Dual-Glo Luciferase Assay System (Promega, Madison, WI, USA). 


\section{Results}

\subsection{FceRI signals induce Mt-1 and Mt-2 expression in basophils}

To determine how Zn-controlling molecules are involved in basophils' FceRI-induced cytokine production, we examined the expression profiles of these molecules in primary basophils before and after FcERI-mediated stimulation by querying the RefDIC (Reference Database of Immune Cells, http://refdic.rcai.riken.jp/). This analysis revealed that Mt-1 and Mt-2 transcription was significantly elevated after stimulation, while that of Zips, ZnTs, Mt-3, and Mt-4 was not affected (Supplemental Fig. S1A-C). The Mt-1 and Mt-2 mRNA levels also increased in primary basophils and BMBAs after lgE-mediated stimulation (Fig. 1A-B). These results indicated that MT-I and MT-II might be involved in basophil functions.

\subsection{MTs are essential for basophils' IgE-mediated IL-4 production}

To assess the involvement of MT-I and MT-II in basophil functions, we analyzed BMBAs prepared from MT-I/II(-/-) mice. We first asked if the basophils developed normally in the absence of MT-I and MT-II. The expression levels of FCERI and CD49b in the MT-I/II(-/-) BMBAs were equivalent to those in wild-type (WT) BMBAs (Fig. 2A). In addition, the expression level of mouse mast cell protease (mMCP-8), a basophil-specific protease (Ugajin et al., 2009), was comparable in MT-I/II(-/-) and WT BMBAs (Fig. 2B). These findings demonstrated that MT-I/II were not required for basophil development. We also 
examined the abilities of MT-I/II(-/-) BMBAs to undergo degranulation and to produce IL-4. MT-I/II(-/-) and WT BMBAs released similar amounts of $\beta$-hexosaminidase in response to Fc\&RI stimulation, indicating that the degranulation in MT-I/II(-/-) BMBAs was normal (Supplemental Fig. S2). However, FceRI induced significantly less IL-4 in MT-I/II(-/-) BMBAs than in WT BMBAs (Fig. 2C, left panel). Quantitative PCR analysis of the II-4 mRNA levels showed that Fc\&RI stimulation increased the II-4 transcript levels in WT BMBAs, and that this increase was significantly diminished in MT-I/II(-/-) BMBAs (Fig. 2C, right panel). These findings indicated that MTs contribute to Fc\&RI-induced IL-4 production at both the mRNA and protein levels in BMBAs.

We next sought to verify the involvement of MTs in the IL-4 production by primary basophils. Blood basophils prepared from WT and MT-I/II(-/-) mice were passively sensitized with TNP-IgE and then stimulated with anti-lgE using an ex vivo basophil stimulation system, as previously described (Charles et al., 2009). FceRI induced significantly less IL-4 from MT-I/II(-/-) than from WT blood basophils (Fig. 2D). These results clearly showed that MTs were required for the Fc\&RI-induced basophil production of IL-4.

\subsection{MTs are selectively required for FceRI-induced CaN/NFAT signaling}

To determine the molecular mechanisms by which MTs regulate basophils' Fc\&RI-induced IL-4 production, we first sought to identify signaling pathways that cause basophils to 
produce IL-4. We first examined the effect of the CaN inhibitor cyclosporine A (CsA) on Fc\&RI-induced IL-4 production. CsA pretreatment significantly diminished the FcERI-stimulated increase in II-4 mRNA (Fig. 3A). The transcription factor NFAT is dephosphorylated by $\mathrm{CaN}$, after which it is translocated to the nucleus to activate target-gene expression (Muller and Rao, 2010). The NFAT family members NFAT1 and NFAT2 were highly expressed in basophils (data not shown). To clarify their roles in basophils' FcERI-mediated IL-4 production, we transfected BMBAs with siRNAs targeting Nfatc2 and Nfatc1 to knock down NFAT1 and NFAT2. We confirmed that the siRNA treatment reduced the Nfatc2 and Nfatc1 mRNA expression (Fig. 3D, left and middle panels). The induction of II-4 mRNA by Fc\&RI was significantly inhibited in cells expressing Nfatc2 or Nfatc1 siRNA (Fig. 3D, right panel). These results indicated that the CaN/NFAT pathway was responsible for the basophils' FceRI-mediated IL-4 production. We also examined whether other signaling molecules, such as PKC and PI3K, were involved in producing IL-4. Go6976 and wortmannin, which inhibit PKC- $\alpha / \beta$ and PI3K, respectively, significantly decreased the amount of $I I-4$ mRNA induced by Fc\&RI stimulation (Fig. 3B-C). Together, these results showed that the PKC, PI3K, and CaN/NFAT signaling pathways were responsible for basophils' Fc\&RI-induced IL-4 production.

To examine whether MTs were involved in any of these signaling pathways responsible for Fc\&RI-induced IL-4 production, we first investigated the involvement of MTs in PKC or PI3K activation. We found that the PKC family members PKC- $\alpha$, PKC- $\beta$, and 
PKC- $\delta$ were expressed in basophils (data not shown). The Fc\&RI-induced translocation of PKC $-\beta$ to the plasma membrane, which is essential for PKC activation, was intact in MT-I/II(-/-) BMBAs (Fig. 4B). The Fc\&RI-induced phosphorylation of Akt, a downstream PI3K target, was also equivalent in WT and MT-I/II(-/-) BMBAs (Fig. 4C). However, the Fc\&RI-induced nuclear translocation of NFAT2 was decreased in MT-I/II(-/-) BMBAs; the frequency of nuclear NFAT2 localization was lower in the MT-I/II(-/-) than in the WT BMBAs

(Fig. 4A). These data indicated that MTs are selectively required for FceRI-induced CaN/NFAT signaling.

\subsection{Elevated intracellular free zinc inhibits CaN activity in MT-I/II(-/-) basophils}

We next sought to identify the upstream targets of MTs involved in NFAT activation, which is known to be regulated by $\mathrm{Ca}^{2+}$ mobilization and the $\mathrm{Ca}^{2+}$-dependent serine/threonine phosphatase CaN (Muller and Rao, 2010). We found that the CaN phosphatase activity was significantly diminished in MT-I/II(-/-) BMBAs (Supplemental Fig. S3B), although the FceRI-induced $\mathrm{Ca}^{2+}$ influx was normal (Supplemental Fig. S3A). Thus, MTs contribute to the nuclear translocation of NFAT in basophils by regulating the $\mathrm{CaN}$ activity.

To further define the molecular mechanism by which MTs regulate Fc\&RI-induced CaN/NFAT signaling, we investigated whether Zn homeostasis was disturbed in MT-I/II(-/-) basophils. For this, we used Newport green DCF diacetate, a cell-permeable Zn-specific fluorescent probe that predominantly localizes to the cytoplasm. We found that the 
intracellular Zn levels were higher in MT-I/II(-/-) than in WT BMBAs (Supplemental Fig. S3C). Intracellular $\mathrm{Zn}$ levels in $100 \mathrm{nM} \mathrm{ZnSO}{ }_{4}$-treated WT BMBAs mimicked those in MT-I/II(-/-) BMBAs (Supplemental Fig. S3D). The phosphatase activity of CaN was diminished in $\mathrm{ZnSO}_{4}$-treated compared to untreated WT BMBAs (Supplemental Fig. S3E). These results supported the idea that MTs impact $\mathrm{CaN}$ activity by regulating the intracellular free $\mathrm{Zn}$ concentration.

\subsection{MT knockdown and reconstitution}

To determine whether the impaired IL-4 production observed in MT-I/II(-/-) basophils was directly caused by the loss of MT function, we knocked down MT-II or both MT-I and MT-II in WT BMBAs by transient siRNA transfection. We confirmed that the siRNA treatment reduced the Mt-1 and Mt-2 mRNA expression (Fig. 5A, left and middle panels). We found that the Fc\&RI-induced IL-4 levels in MT-II single-knockdown and MT-I/II double-knockdown BMBAs were significantly lower than in control BMBAs (Fig. 5A, right panel and 5B). We further confirmed that transfecting MT-I/II(-/-) basophils with MT-II cDNA rescued the defective Fc\&RI-induced IL-4 production. In this experiment, the BMBAs were transiently co-transfected with a murine IL-4 promoter luciferase construct, a renilla luciferase control reporter, and either MT-II cDNA or an empty vector. The FceRI-induced increase in IL-4 promoter-driven luciferase expression, which was observed in mock-transfected WT BMBAs, was significantly diminished in the mock-transfected 
MT-I/II(-/-) BMBAs (Fig. 5C), consistent with the impaired IL-4 production observed in MT-I/II(-/-) BMBAs (Fig. 2C). MT-II overexpression in the MT-I/II(-/-) BMBAs restored the FceRI-induced IL-4-promoter-driven luciferase activity to normal levels (Fig. 5C). Taken together, these findings showed that MTs are required for regulating the Fc\&RI-induced IL-4 production in basophils. 


\section{Discussion}

Our previous studies revealed that $\mathrm{Zn}$ acts as an essential signaling molecule in Fc\&RI-stimulated mast cell activation and cytokine production in mouse (Kabu et al., 2006; Nishida et al., 2009; Yamasaki et al., 2012). However, Zn's role in basophil activation has been unclear. Here, we provide evidence that both $\mathrm{Zn}$ and the $\mathrm{Zn}$-binding MT proteins are involved in the Fc\&RI-induced IL-4 production in basophils.

$\mathrm{Zn}$ homeostasis is maintained by its transport through fourteen ZIP-family and eight $\mathrm{ZnT}$-family members and by its buffering by four MT-family members. All of these protein families play a role in the intricate regulation of free $\mathrm{Zn}$, and the members involved may depend on the cell type. In mast cells, the ZnT5 expression is up-regulated by FceRI stimulation, and ZnT5 is required for the FceRI-induced mast cell cytokine production that occurs through NF-kB activation (Nishida et al., 2009). In the present study, we showed that Fc\&RI stimulation increased the expression levels of MT-I and MT-II among the Zn-regulating molecules in basophils (Supplemental Fig. S1A-C), suggesting that MT-dependent $\mathrm{Zn}$ regulation is involved in basophil function downstream of Fc\&RI signaling. In fact, our results showed that MTs were involved in the Fc\&RI-induced IL-4 production but not in degranulation in basophils (Fig. 2C-D and Supplemental Fig. S2).

Basophils are known to produce large amounts of Th2-promoting cytokines, including IL-4 and TSLP (Schroeder et al., 2001; Seder et al., 1991; Sokol et al., 2008; 
Voehringer et al., 2004). However, the molecular mechanisms of basophil IL-4 production have not been fully elucidated. Here, using pharmacological inhibitors and RNA-interference experiments, we identified three regulatory pathways responsible for FceRI-induced IL-4 production: CaN/NFAT signaling, PKC signaling, and PI3K/Akt signaling.

There are four or five NFAT-binding sites in the mouse and human IL-4 promoters (Serfling et al., 2000). Of the five NFAT-family members (NFAT1-5), NFAT2 is the most predominantly expressed in human basophils (Schroeder et al., 2002). Fc\&RI signals induce NFAT2 accumulation in the nucleus and increase NFAT's binding to the IL-4 promoter (Qi et al., 2011; Schroeder et al., 2002), suggesting that NFAT2 may be required for IgE-mediated basophil IL-4 production. In the present study, treatment with either Nfatc2 (NFAT1) or Nfatc1 (NFAT2) siRNA inhibited the FceRI-induced increase in II-4 mRNA in BMBAs (Fig. 3D).

In addition, the $\mathrm{CaN}$ inhibitor $\mathrm{CsA}$ abolished the Fc\&RI-mediated induction of II-4 mRNA in BMBAs (Fig. 3A). Our findings demonstrated that CaN/NFAT signaling is required for Fc\&RI-mediated IL-4 production by mouse basophils. In mast cells, PKC is responsible for Fc\&RI-induced cytokine production through NF-кB activation (Kabu et al., 2006; Nechushtan et al., 2000). We found that the PKC $\alpha / \beta$ inhibitor Go6976 attenuated the FceRI-stimulated II-4 induction in BMBAs (Fig. 3B), suggesting that PKC positively regulates basophils' Fc\&RI-induced IL-4 production. 
$\mathrm{PI} 3 \mathrm{~K}$ is reported to be required for $\mathrm{IgE}$-induced mediator release from human basophils (Gibbs and Grabbe, 1999; Miura and MacGlashan, 2000). We found that the PI3K inhibitor wortmannin also attenuated the BMBAs' FceRI-induced IL-4 production (Fig. $3 \mathrm{C}$ ), indicating that $\mathrm{PI} 3 \mathrm{~K}$ activation is required for this IL-4 production in both mouse and human basophils. Thus, the signaling pathways responsible for Fc\&RI-induced IL-4 production are conserved, and are mediated by the activation of PKC and PI3K and by CaN/NFAT signaling.

Since our results showed that MTs are required for basophils' IL-4 production, we next asked which of the above pathways is regulated by MTs. The FceRI-mediated nuclear translocation of NFAT was impaired in MT-I/II(-/-) BMBAs (Fig. 4A), while both the Fc\&RI-induced translocation of PKC $\beta$ to the membrane and the phosphorylation of PI3K's downstream target Akt were intact in these cells (Fig. 4B-C). Therefore, MTs are selectively required for Fc\&RI-induced CaN/NFAT signaling. Ca mobilization activates calmodulin and calmodulin-dependent CaN, thereby inducing NFAT's nuclear translocation (Muller and Rao, 2010). Ca mobilization was intact in MT-I/II(-/-) BMBAs (Supplemental Fig. S3A), consistent with our finding that the degranulation of MT-I/II(-/-) BMBAs was normal (Supplemental Fig. S2). However, CaN's phosphatase activity was significantly impaired in MT-I/II(-/-) BMBAs, despite being expressed at normal levels (Supplemental Fig. S3B and data not shown). These results indicated that MTs regulate CaN's phosphatase activity independently of $\mathrm{Ca}$ signaling. 
$\mathrm{CaN}$ is a heterodimer consisting of a catalytic subunit, $\mathrm{CaN} \mathrm{A}$, and a regulatory subunit, $\mathrm{CaN} B$. The catalytic domain contains an $\mathrm{Fe}^{3+}-\mathrm{Zn}^{2+}$ dinuclear center, and $\mathrm{Zn}$ is thought to act as a catalytic cofactor (King and Huang, 1984; Rusnak and Mertz, 2000). On the other hand, $\mathrm{Zn}$ is reported to inhibit CaN's activity in vitro (Aydemir et al., 2009; Huang et al., 2008; Takahashi et al., 2003). In the current study, the intracellular free $\mathrm{Zn}$ concentration was higher in MT-I/II(-/-) than in WT BMBAs (Supplemental Fig. S3C) due to the absence of MTs, which are important for storing cytosolic Zn. Experimentally increased Zn levels attenuated the CaN activity in WT BMBAs (Supplemental Fig. S3E). Together, these findings suggest that the MT-mediated regulation of intracellular Zn levels plays an important role in modulating the CaN/NFAT signaling in basophils.

In summary, the present study revealed a novel molecular mechanism that regulates the basophil production of IL-4, involving Zn's sequestration by MTs. This finding suggests that the MTs could be key therapeutic targets for basophil-mediated immune responses and allergic inflammation. 


\section{Conflict of interest statement}

The authors have no conflicts of interest to declare.

\section{Acknowledgements}

We thank A. Ito and C. Miyagishi for technical assistance, Drs. T. Kambe, S. Himeno, and P.

Burrows for helpful discussion, the IMS-RCAI FACS Laboratory for cell sorting, R. Kimura

for secretarial assistance, and the IMS-RCAI animal facility for breeding and maintaining

the mice for this study.

This work was partly supported by Grants-in-Aid for Scientific Research (KAKENHI \#22890054, \#22591218 \#23590576, \#24249028), the JST-CREST Program, the Takeda

Science Foundation (K.N.), the Mochida Memorial Foundation for Medical and

Pharmaceutical Research (K.N.), TMDU Presidential Grant-in-Aid for Young Scientists (T.U.), the Suzuken Memorial Foundation (S.Y.), and a grant from the Ministry of Health, Labor and Welfare (H-24-007), Japan (H.Y.). 


\section{References}

Andrews G.K. (2001) Cellular zinc sensors: MTF-1 regulation of gene expression. Biometals 14, 223-237.

Aydemir T.B., Liuzzi J.P., McClellan S., Cousins R.J. (2009) Zinc transporter ZIP8 (SLC39A8) and zinc influence IFN-gamma expression in activated human T cells. J. Leukoc. Biol. 86, 337-348.

Charles N., Watford W.T., Ramos H.L., Hellman L., Oettgen H.C., Gomez G., Ryan J.J., O'Shea J.J., Rivera J. (2009) Lyn kinase controls basophil GATA-3 transcription factor expression and induction of Th2 cell differentiation. Immunity 30, 533-543.

Fukada T., Yamasaki S., Nishida K., Murakami M., Hirano T (2011). Zinc homeostasis and signaling in health and diseases: Zinc signaling. J. Biol. Inorg. Chem. 16, 1123-34.

Galli S.J. (2000) Mast cells and basophils. Curr. Opin. Hematol. 7, 32-9.

Gibbs B.F., Grabbe J. (1999) Inhibitors of PI 3-kinase and MEK kinase differentially affect mediator secretion from immunologically activated human basophils. J. Leukoc. Biol. 65, 883-90.

Hirano T., Murakami M., Fukada T., Nishida K., Yamasaki S., Suzuki T. (2008) Roles of zinc and zinc signaling in immunity: zinc as an intracellular signaling molecule. Adv. Immunol. 97, 149-76.

Huang J., Zhang D., Xing W., Ma X., Yin Y., Wei Q., Li G. (2008) An approach to assay calcineurin activity and the inhibitory effect of zinc ion. Anal. Biochem. 375, 385-7.

Kabu K., Yamasaki S., Kamimura D., Ito Y., Hasegawa A., Sato E., Kitamura H., Nishida K., Hirano T. (2006) Zinc is required for Fc epsilon RI-mediated mast cell activation. J. Immunol. 177, 1296-305.

Karasuyama H., Yamanishi Y. (2014) Basophils have emerged as a key player in immunity. Curr. Opin. Immunol. 31, 1-7.

King M.M., Huang C.Y. (1984) The calmodulin-dependent activation and deactivation of the phosphoprotein phosphatase, calcineurin, and the effect of nucleotides, pyrophosphate, and divalent metal ions. Identification of calcineurin as a $\mathrm{Zn}$ and Fe metalloenzyme. J. Biol. Chem. $259,8847-56$.

Kubo M., Kincaid R.L., Webb D.R., Ransom J.T. (1994) The Ca2+/calmodulin-activated, phosphoprotein phosphatase calcineurin is sufficient for positive transcriptional regulation of the mouse IL-4 gene. Int. Immunol. 6, 179-88.

Macfarlane A.J., Kon O.M., Smith S.J., Zeibecoglou K., Khan L.N., Barata L.T., McEuen A.R., Buckley M. G., Walls A.F., Meng Q., Humbert M., Barnes N.C., Robinson D.S., Ying S., Kay A.B. (2000) Basophils, eosinophils, and mast cells in atopic and nonatopic asthma and in late-phase allergic reactions in the lung and skin. J. Allergy Clin. Immunol. 105, 99-107.

Miura K., MacGlashan D.W. (2000) Phosphatidylinositol-3 kinase regulates p21ras activation during IgE-mediated stimulation of human basophils. Blood 96, 2199-205.

Muller M.R., Rao A. (2010) NFAT, immunity and cancer: a transcription factor comes of age. Nat. Rev. Immunol. 10, 645-56. 
Nechushtan H., Leitges M., Cohen C., Kay G., Razin E. (2000) Inhibition of degranulation and interleukin -6 production in mast cells derived from mice deficient in protein kinase Cbeta. Blood $95,1752-7$.

Nishida K., Hasegawa A., Nakae S., Oboki K., Saito H., Yamasaki S., Hirano T. (2009) Zinc transporter Znt5/Slc30a5 is required for the mast cell-mediated delayed-type allergic reaction but not the immediate-type reaction. J. Exp. Med. 206, 1351-64.

Palmiter R.D. (1998) The elusive function of metallothioneins. Proc. Natl. Acad. Sci. US A 95, 8428-30.

Palmiter R.D. (2004) Protection against zinc toxicity by metallothionein and zinc transporter 1. Proc. Natl. Acad. Sci. U S A 101, 4918-23.

Plager D.A., Weiss E.A., Kephart G.M., Mocharla R.M., Matsumoto R., Checkel J.L., Schwartz L.B., Gleich G.J., Leiferman K.M. (2006) Identification of basophils by a mAb directed against pro-major basic protein 1. J. Allergy Clin. Immunol. 117, 626-34.

Prasad A.S. (1995) Zinc: an overview. Nutrition 11, 93-9.

Qi X., Nishida J., Chaves L., Ohmori K., Huang H. (2011) CCAAT/enhancer-binding protein alpha (C/EBPalpha) is critical for interleukin-4 expression in response to FcepsilonRI receptor cross-linking. J. Biol. Chem. 286, 16063-73.

Rusnak F., Mertz P. (2000) Calcineurin: form and function. Physiol. Rev. 80, 1483-521.

Schroeder J.T., MacGlashan D.W., Jr., Lichtenstein L.M. (2001) Human basophils: mediator release and cytokine production. Adv. Immunol. 77, 93-122.

Schroeder J.T., Miura K., Kim H. H., Sin A., Cianferoni A., Casolaro V. (2002) Selective expression of nuclear factor of activated $\mathrm{T}$ cells $2 / \mathrm{c} 1$ in human basophils: evidence for involvement in IgE-mediated IL-4 generation. J. Allergy Clin. Immunol. 109, 507-13.

Seder R.A., Paul W.E., Dvorak A.M., Sharkis S.J., Kagey-Sobotka A., Niv Y., Finkelman F.D., Barbieri S. A., Galli S.J., Plaut M. (1991) Mouse splenic and bone marrow cell populations that express high-affinity Fc epsilon receptors and produce interleukin 4 are highly enriched in basophils. Proc. Natl. Acad. Sci. U S A 88, 2835-9.

Serfling E., Berberich-Siebelt F., Chuvpilo S., Jankevics E., Klein-Hessling S., Twardzik T., Avots A. (2000) The role of NF-AT transcription factors in T cell activation and differentiation. Biochim. Biophys. Acta 1498, 1-18.

Sokol C.L., Barton G.M., Farr A.G., Medzhitov R. (2008) A mechanism for the initiation of allergen-induced T helper type 2 responses. Nat. Immunol. 9, 310-8.

Takahashi K., Akaishi E., Abe Y., Ishikawa R., Tanaka S., Hosaka K., Kubohara Y. (2003) Zinc inhibits calcineurin activity in vitro by competing with nickel. Biochem. Biophys. Res. Commun. 307, 64-8.

Thirumoorthy N., Shyam Sunder A., Manisenthil Kumar K., Senthil Kumar M., Ganesh G., Chatterjee M. (2011) A review of metallothionein isoforms and their role in pathophysiology. World J. Surg. Oncol. 9, 54 .

Ugajin T., Kojima T., Mukai K., Obata K., Kawano Y., Minegishi Y., Eishi Y., Yokozeki H., Karasuyama H. (2009) Basophils preferentially express mouse mast cell protease 11 among the mast cell 
tryptase family in contrast to mast cells. J. Leukoc. Biol. 86, 1417-25

Ugajin T., Satoh T., Kanamori T., Aritake K., Urade Y., Yokozeki H. (2011) FcepsilonRI, but not FcgammaR, signals induce prostaglandin D2 and E2 production from basophils. Am. J. Pathol. $179,775-82$.

Voehringer D., Shinkai K., Locksley R.M. (2004) Type 2 immunity reflects orchestrated recruitment of cells committed to IL-4 production. Immunity 20, 267-77.

Yamasaki S., Hasegawa A., Hojyo S., Ohashi W., Fukada T., Nishida K., Hirano T. (2012) A novel role of the L-type calcium channel alpha1D subunit as a gatekeeper for intracellular zinc signaling: zinc wave. PLoS One 7, e39654.

Yamasaki S., Sakata-Sogawa K., Hasegawa A., Suzuki T., Kabu K., Sato E., Kurosaki T., Yamashita S., Tokunaga M., Nishida K., Hirano T. (2007) Zinc is a novel intracellular second messenger. J. Cell Biol. 177, 637-45. 


\section{Figure legends}

Figure 1. Fc\&RI-mediated stimulation up-regulates MT expression. BMBAs $(A)$ and primary basophils $\left(\mathrm{CD} 49 \mathrm{~b}^{+} \mathrm{CD} 123^{+}\right.$cells) $(\mathrm{B})$ isolated from the bone marrow cells of C57BL6/J mice were sensitized with $1 \mu \mathrm{g} / \mathrm{ml}$ anti-TNP-lgE and stimulated with $100 \mathrm{ng} / \mathrm{ml}$ TNP-OVA for the indicated times. Gene expression levels were measured using SYBR Green Dye with a real-time PCR system, and were normalized to Gapdh. The data show the mean \pm SD. ${ }^{*} P<0.05 ;{ }^{* *} P<0.01$, Student's $t$-test. Datasets are representative of three (A, B) experiments.

Figure 2. MT-I/II(-/-) basophils have impaired IL-4 production. (A) BMBAs from WT and MT-I/II(-/-) mice were identified as $\mathrm{CD} 49 \mathrm{~b}^{+} \mathrm{Fc \varepsilon RI^{+ }}$ cells by flow cytometric analysis. (B) Cytospin slides prepared from WT and MT-I/II(-/-) BMBAs were stained with an anti-mMCP-8 mAb (green) and DAPI (blue). Scale bar, $20 \mu \mathrm{m}$. The percentage of mMCP-8-positive cells is shown below the photomicrograph. Data show the mean+SD. N.S., not significant, Student's t-test. (C) BMBAs from WT (open bars) or MT-I/II(-/-) (closed bars) mice were sensitized with $1 \mu \mathrm{g} / \mathrm{ml}$ anti-TNP-IgE and stimulated with the indicated TNP-OVA concentrations for the indicated times. IL-4 concentrations in the supernatants were determined by ELISA (left panel). II-4 transcript levels were measured by real-time PCR and normalized to Gapdh (right panel). (D) Blood basophils from WT (open bars) and 
MT-I/II(-/-) (closed bars) mice ( $n=3)$ were passively sensitized with TNP-IgE and stimulated with anti-lgE using a previously described ex vivo basophil-stimulation system (Charles et al., 2009). Plasma IL-4 levels were determined by ELISA. Data show the mean \pm SD.

${ }^{*} P<0.05$ or ${ }^{\star \star} P<0.01$ by Student's $t$-test, compared with WT basophils. Datasets are representative of three experiments.

Figure 3. FceRI-induced IL-4 production is dependent on Calcineurin/NFAT, PKC, and PI3K-mediated pathways.

(A) BMBAs sensitized with $1 \mu \mathrm{g} / \mathrm{ml}$ anti-TNP-lgE were treated with $10 \mu \mathrm{M}$ cyclosporin A (CsA) (closed bars) or vehicle (open bars) for $20 \mathrm{~min}$, then stimulated with $100 \mathrm{ng} / \mathrm{ml}$ TNP-OVA for $1 \mathrm{~h}$. (B and C) BMBAs sensitized with $1 \mu \mathrm{g} / \mathrm{ml}$ anti-TNP-IgE were treated with $5 \mu \mathrm{M}$ Go6976 (B, closed bars), $100 \mathrm{nM}$ Wortmannin (C, closed bars), or vehicle (open bars) for $2 \mathrm{~h}$, then stimulated with $100 \mathrm{ng} / \mathrm{ml}$ TNP-OVA (Ag) or nothing (Non) for $1 \mathrm{~h}$. IL-4 expression at the mRNA level was assessed by real-time quantitative RT-PCR. (D) NFAT1-knockdown, NFAT2-knockdown, and control BMBAs were sensitized with $1 \mu \mathrm{g} / \mathrm{ml}$ TNP-IgE, followed by stimulation with $100 \mathrm{ng} / \mathrm{ml}$ TNP-OVA (Ag) or nothing (Non) for $1 \mathrm{~h}$. Gene expression levels were assessed by real-time quantitative RT-PCR. Data show the mean+SD. ${ }^{*} p<0.05,{ }^{* *} p<0.01$, compared with vehicle-treated or control BMBAs, Student's $t$-test. A representative dataset of three independent experiments, each which gave similar results, is shown. 


\section{Figure 4. MT-I/II(-/-) BMBAs have defective FceRI-mediated CaN/NFAT signaling.}

WT and MT-I/II(-/-) BMBAs were stimulated with $100 \mathrm{ng} / \mathrm{ml}$ TNP-OVA (Ag) or nothing (Non) for $30 \mathrm{~min}(\mathrm{~A})$ or $10 \mathrm{~min}(\mathrm{~B}, \mathrm{C})$. (A) Cells were stained with an anti-NFAT2 Ab (green) and DAPI (4,6 diamidino-2-phenylindole) (blue). Scale bar, $10 \mu \mathrm{m}$. The frequency of cells with nuclear NFAT2 staining is shown at right. For each experiment, 100 cells were randomly counted, and the percentage of cells with translocated nuclear NFAT2 was calculated. (B) Cells were stained with an anti-PKC- $\beta$ Ab (green) and DAPI (blue). Scale bar, $10 \mu \mathrm{m}$. The percentage of cells showing PKC- $\beta$ translocation to the plasma membrane is shown at right. For each experiment, 100 randomly counted cells were analyzed. (C) Cell lysates were immunoblotted with anti-phospho Akt, -Akt, and -actin mAbs. Densitometric values are shown at right. Data show the mean \pm SD. ${ }^{*} P<0.05$, compared with WT BMBA, Student's $t$-test. Datasets shown are representative of three experiments.

\section{Figure 5. Knockdown and reconstitution of MTs.}

(A and B) MT-II knockdown (gray bars), MT-I/II double knockdown (closed bars), and control (open bars) BMBAs were sensitized with $1 \mu \mathrm{g} / \mathrm{ml}$ TNP-lgE, followed by stimulation with $100 \mathrm{ng} / \mathrm{ml}$ TNP-OVA (Ag) or nothing (Non) for $1 \mathrm{~h}$ (A, right panel) or $3 \mathrm{~h}(\mathrm{~B})$. Gene expression levels were assessed by real-time quantitative RT-PCR (A). The IL-4 concentration in the supernatants was determined by ELISA (B). Data show the mean+SD. 
${ }^{*} \mathrm{p}<0.05,{ }^{* *} \mathrm{p}<0.01$, compared with control BMBAs. (C) WT and MT-I/II(-/-) BMBAs were transfected with pGL4-IL-4-Luc and phRL-TK together with vectors expressing MT-II or Mock. The cells were then sensitized with $1 \mu \mathrm{g} / \mathrm{ml}$ TNP-lgE, followed by stimulation with $100 \mathrm{ng} / \mathrm{ml}$ TNP-OVA (Ag) or nothing (Non) for $2 \mathrm{~h}$. The normalized luciferase activity obtained from non-stimulated cells was set as 1 . Data show the mean $+S D .{ }^{*} p<0.05$, ${ }^{* *} p<0.01$, Student's $t$-test. Datasets shown are representative of three experiments. 

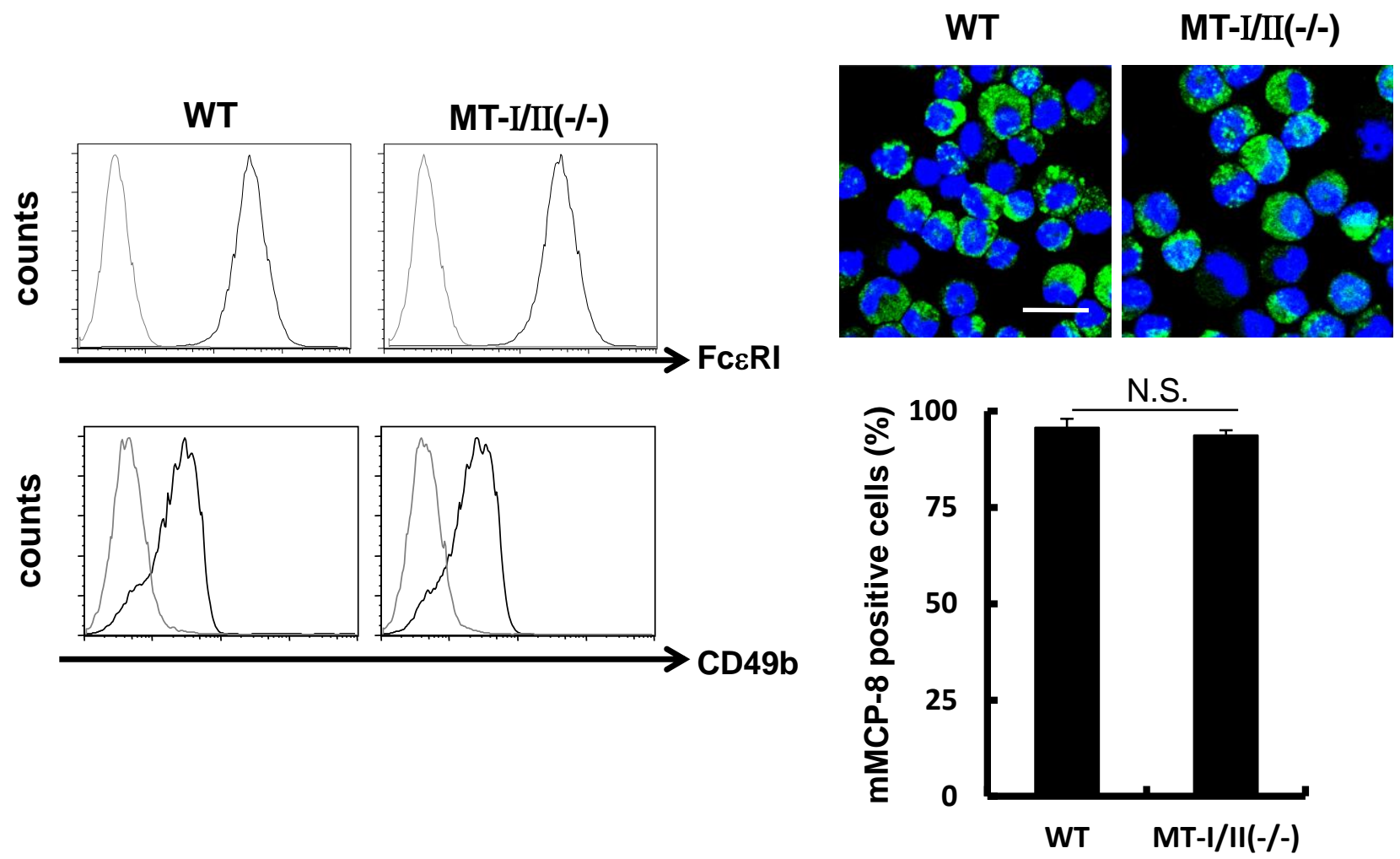

C
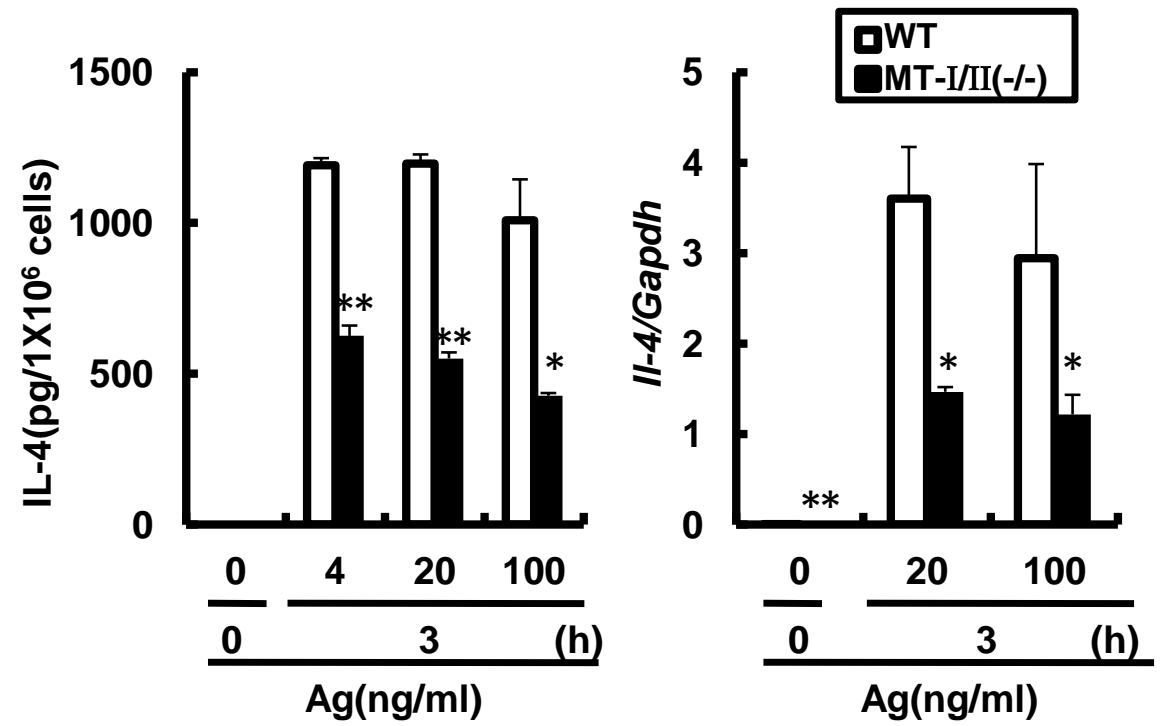

D

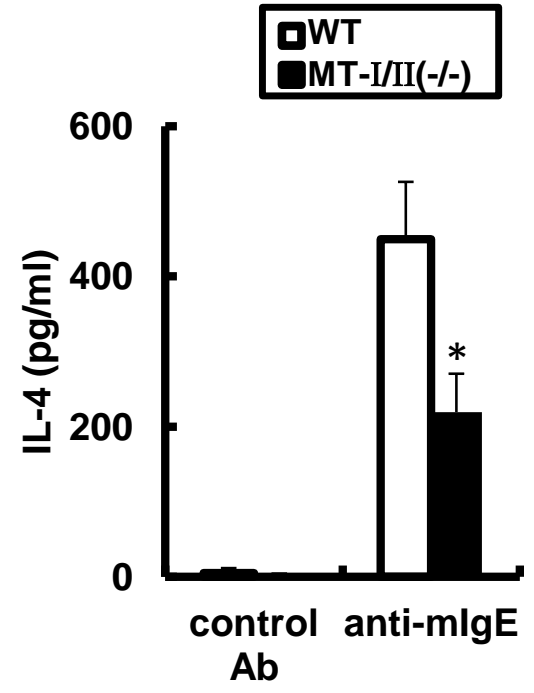

Figure 2 
A

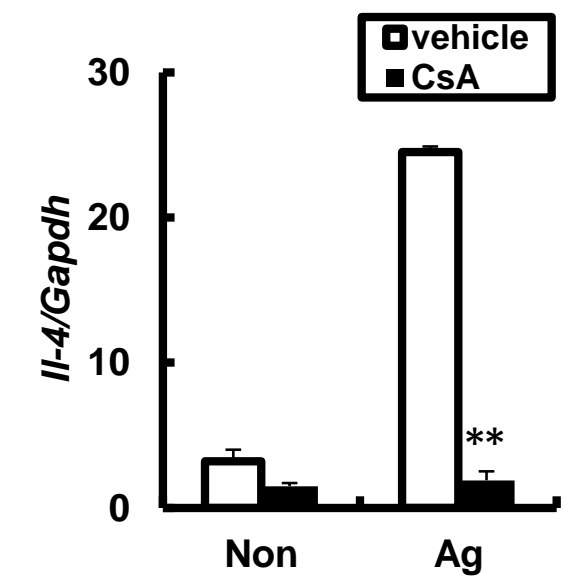

D

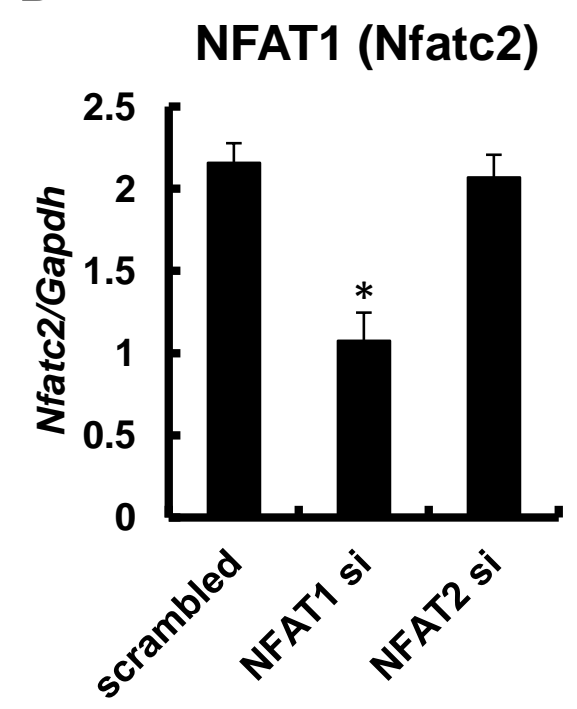

B
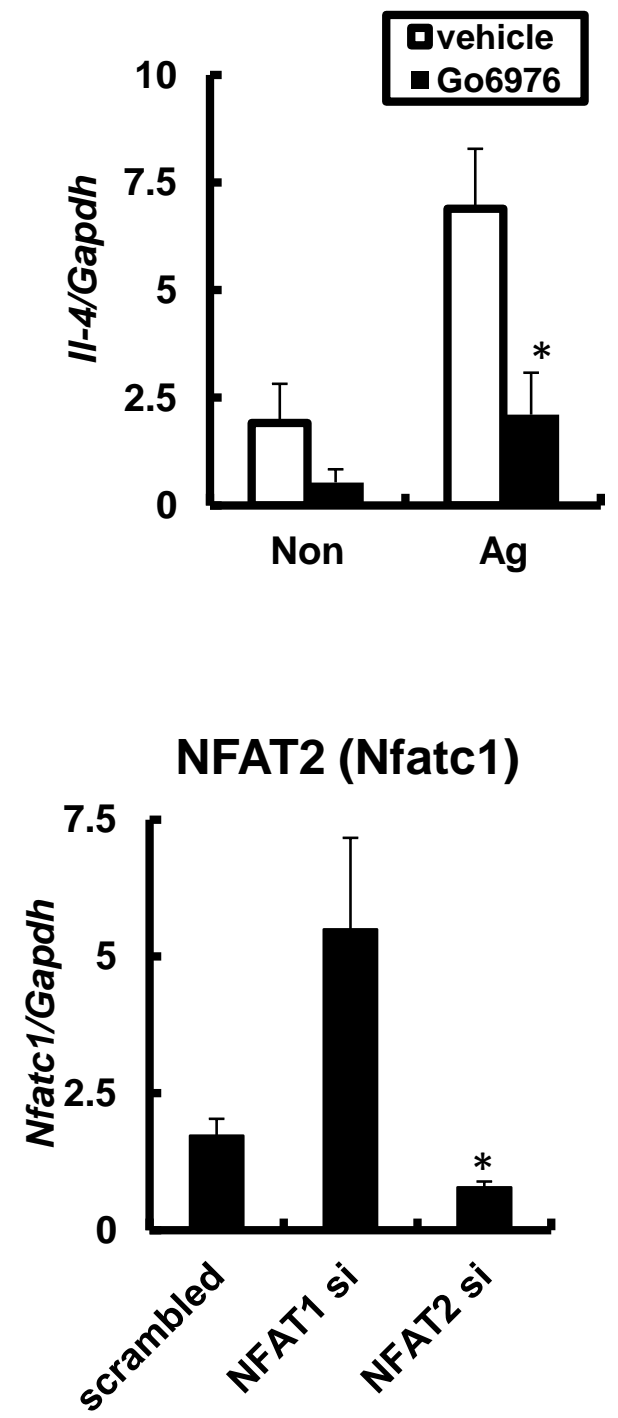

C
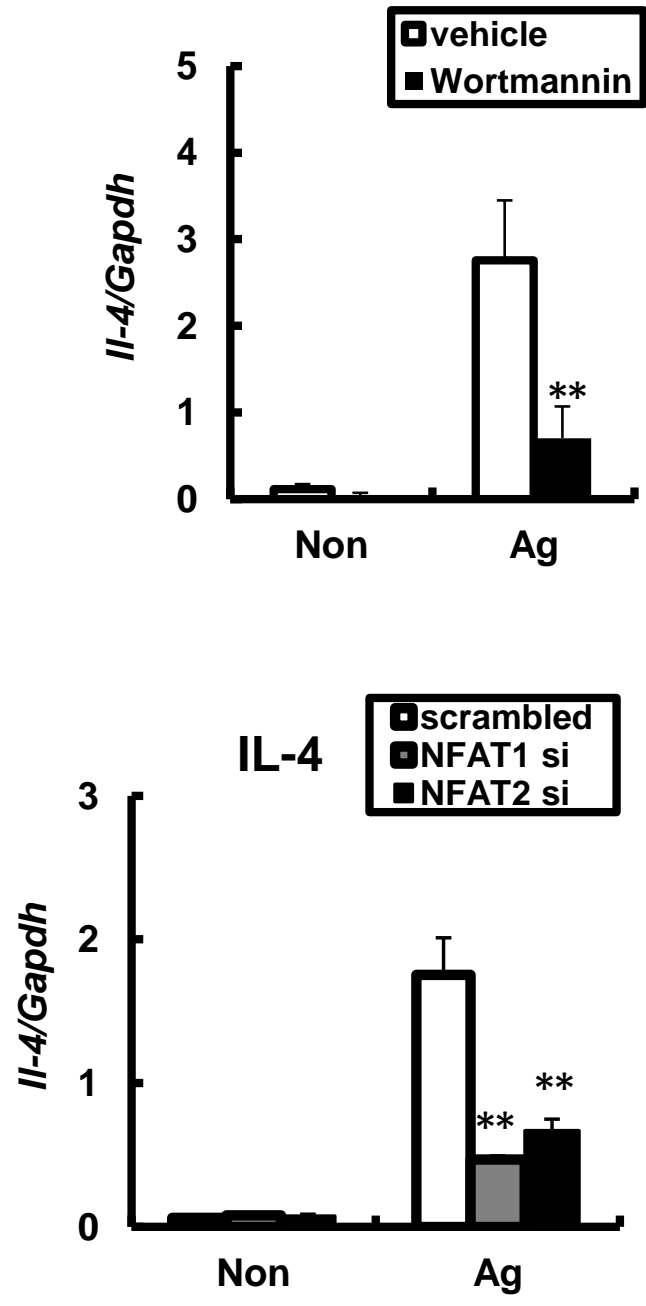
A
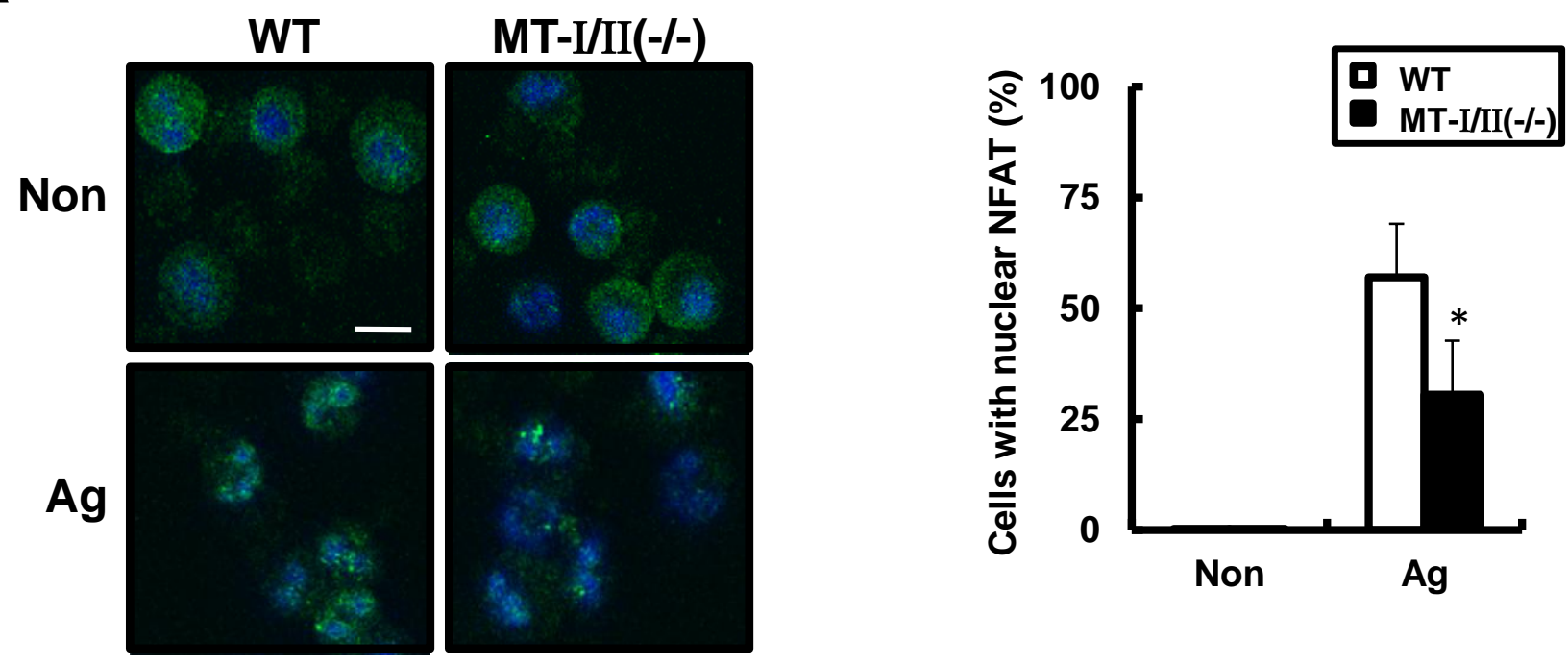

B
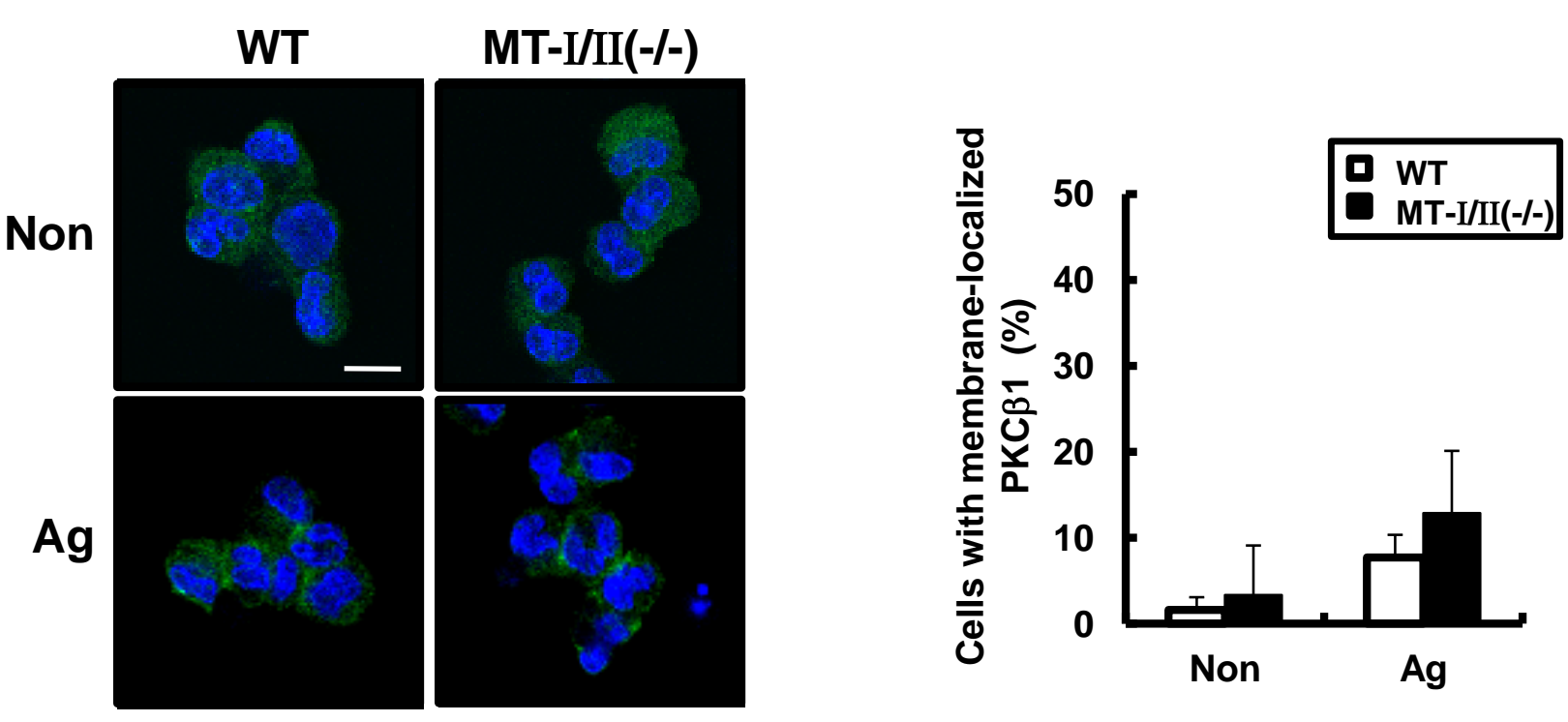

C
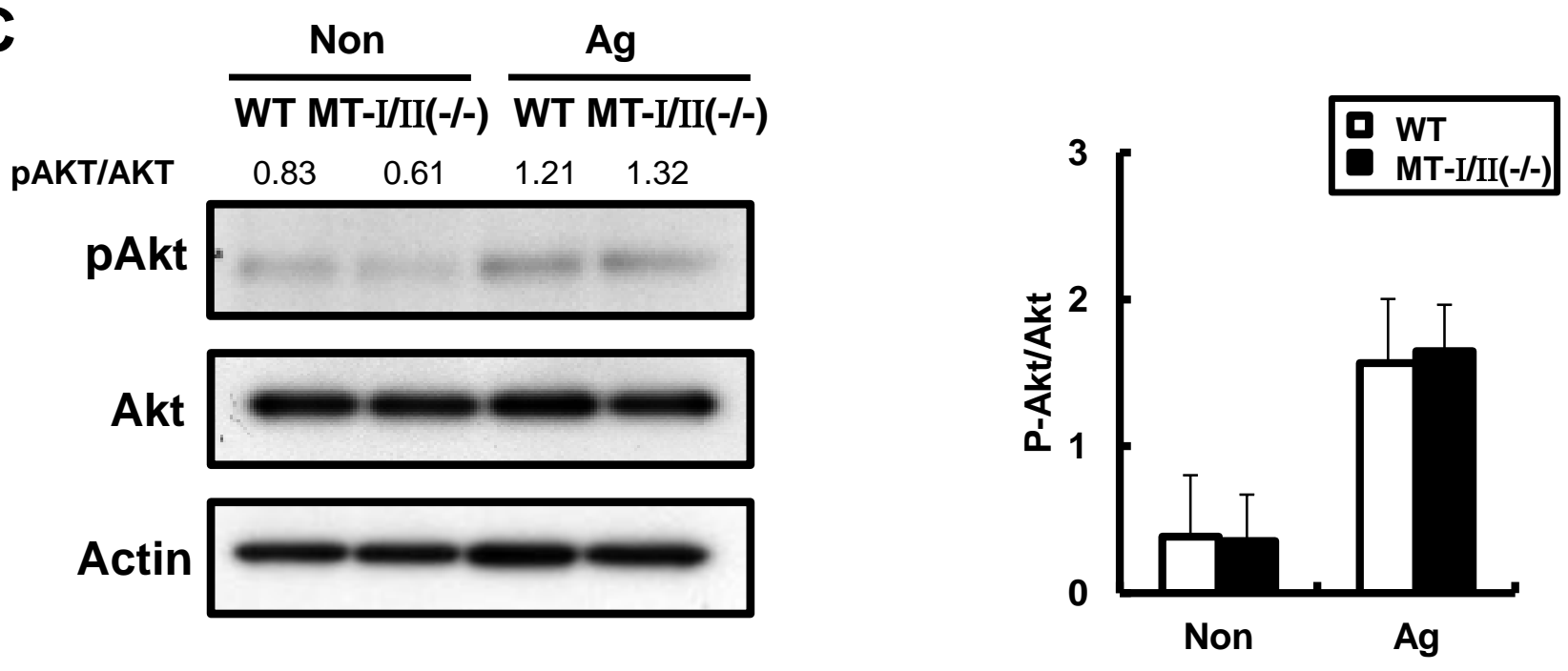

\section{Figure 4}



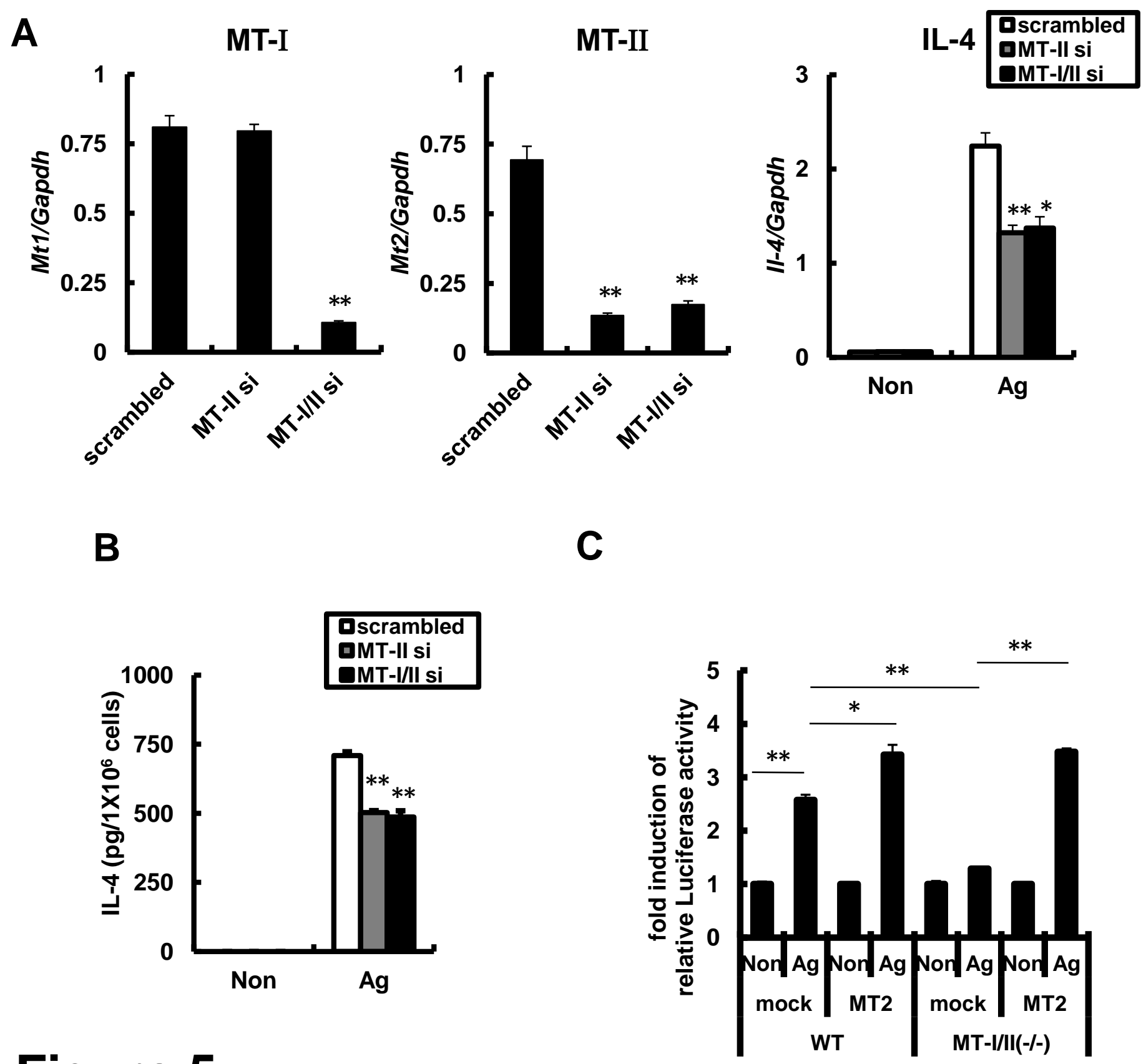

\section{Figure 5}

